\title{
Efficacy of surgical helmet systems for protection against COVID-19: a double-blinded randomised control study
}

\author{
Gavin Schaller ${ }^{1}$ (D) Sandeep Krishan Nayar ${ }^{1} \cdot$ Marios Erotocritou $^{1} \cdot$ Alexander Overton $^{1} \cdot$ Thomas Stelzhammer $^{1}$. \\ Onur Berber ${ }^{1}$
}

Received: 18 June 2020 / Accepted: 1 September 2020 / Published online: 8 September 2020

(C) SICOT aisbl 2020

\begin{abstract}
Purpose This study assesses whether sterile surgical helmet systems (SSHS) provide surgeons with additional protection from aerosol pathogens alongside their traditional role protecting against splash. There has been debate on whether to use such systems in reopening elective orthopaedic surgery during the current COVID-19 pandemic environment.

Methods Thirty-five participants were enrolled in a double-blinded randomised controlled study investigating efficacy of the Stryker Flyte Surgical Helmet (Stryker Corporation, Kalamazoo, MI, USA) as protection against respiratory droplets. Wearing the SSHS in a fit testing hood, subjects were randomised to nebulised saccharin solution or placebo. Twenty were allocated to the saccharin group with 15 to placebo. Positive sweet taste represented a failure of the test. Taste tests were performed with the helmet fan turned on and off.

Results SSHS did not prevent saccharin taste $(p<0.0001)$. Within the saccharin cohort, $40 \%$ recorded a positive taste with the fan on and $100 \%$ with the fan off. There was a statistically significant difference in mean time-to-taste saccharin $(p=0.049)$ comparing fan on (123.5 s) vs. off (62.6 s).

Conclusions SSHS do not protect against aerosol particulate and therefore are not efficacious in protection against COVID-19. The fan system employed may even increase risk to the surgeon by drawing in particulates as well as delay recognition of intraoperative cues, such as exhaust from diathermy, that point to respirator mask leak.
\end{abstract}

Keywords PPE $\cdot$ Sterile surgical helmet systems $\cdot$ COVID-19

\section{Introduction}

The pandemic caused by the severe acute respiratory syndrome coronavirus 2 (SARS-CoV-2), also known as COVID-19, continues to have unprecedented impact across the globe [1]. COVID-19 is highly contagious and is predominantly spread via respiratory droplets [2]. One widely reported concern has been the availability and use of personal protective equipment (PPE) [3]. International guidance in performing aerosol generating procedures is to wear a longsleeved fluid repellent disposable gown, a fit checked respirator mask (such as the N95 or filtering facepiece 3 [FFP3]), eye protection and gloves [4-6].

Gavin Schaller

Gavin.schaller@nhs.net

1 Trauma and Orthopaedics Department, Whittington Health NHS Trust, Magdala Avenue, London N19 5NF, UK
Prior to the COVID-19 outbreak, sterile surgical helmet systems (SSHS), such as the Stryker Flyte and T4/T5 togas and hood (Stryker Corporation, Kalamazoo, MI, USA), were used in elective total hip and knee arthroplasty [7, 8]. SSHS cover the entire head and incorporate a head-mounted fan, drawing in air from outside the hood to inside the face shield and then circulating it out through the bottom of the surgical gown towards the operating room floor [9]. The Stryker Flyte Surgical Helmet system has a filter over the head-mounted fan. Air passes through an Association for the Advancement of Medical Instrumentation (AAMI) class 3 material on the exterior before passing through a AAMI class 4 material inside the hood [10,11]. The class 4 material offers the highest level of protection against pathogens; however, this is based on direct contact with a liquid [8]. The SSHS are used to try and reduce microorganism spread from the surgeon to patient, reduce the risk of surgical site infection and protect the surgeon from any gross contamination such as blood splashes [7]. Whilst studies have not demonstrated a clear benefit to SSHS in reducing deep infections in patients post arthroplasty 
surgery, they are still used internationally, and many surgeons use them for the enhanced splash protection they afford [7]. The helmet systems with the head-mounted fan and battery are non-sterile and can be stored at room temperature, suitable for immediate reuse. These are covered with a disposable sterile hood and visor prior to intraoperative use. It is this sterile hood that contains the AAMI 3 and 4 filters which sit directly over the fan. The International Consensus Group recently advocated the use of the SSHS if they are mandated by hospital standard protocols for total joint arthroplasty [12]. During the ongoing pandemic, there has been reported use of these systems as additional protection against spread of the virus from aerosol generating procedures, used alongside respirator masks. This is despite the systems not being validated for use in this context in aerosol contact and not being recommended for this purpose by the manufacturers $[12,13]$.

The primary goal of this study was to investigate whether surgical helmet systems can be considered protective against COVID-19 in the current pandemic climate, with relevance to the growing discussion over how to safely undertake elective orthopaedic surgery.

\section{Materials and methods}

\section{Null hypothesis}

Sterile surgical helmet systems provide protection against respiratory droplet spread.

\section{Study design}

A double-blinded randomised controlled study was undertaken investigating the efficacy of the Stryker Flyte Surgical Helmet (Stryker Corporation, Kalamazoo, MI, USA) as protection against respiratory droplets. The helmet systems were tested using a validated qualitative test for respirator masks, whereby the participant is exposed to an aerosolised saccharin solution protocol whilst wearing the apparatus, and a positive taste result indicates failure of the equipment $[14,15]$. This was carried out in accordance with standards set by international guidance [16, 17].

Participant eligibility criteria were adults (greater than 18 years) who were healthy as determined by the investigator, with no existing comorbidities that may impact the ability to taste or smell, and capable of giving informed consent.

Saccharin taste tests were performed using the $3 \mathrm{M}^{\mathrm{TM}}$ Qualitative Fit Test Apparatus on all participants. Prior to commencement of formal testing, participants underwent a saccharin taste threshold screening test to ensure sensitivity to the saccharin solution.

Following this taste test, all candidates were given five minute to clear the taste from their mouth. Candidates were then placed in the Stryker Flyte Surgical Helmet and a 3M $\mathrm{M}^{\mathrm{TM}}$ FT-10 Fit Test Hood was placed over the top allowing the atmosphere around the helmet to be controlled.

Participants were subsequently randomised to be exposed to either a nebulised $45 \%$ sodium saccharin solution or a placebo (sterilised water). Both nebulised solutions were blinded to the observer and participant. Each test was undertaken over a maximum of five minutes. In order to further evaluate whether air clearance had any effect on the results, the test was performed twice at each sitting, first with the helmet system's fan on maximum and then with the fan turned off. Outcome measures were whether saccharin was tasted, and if so, the time taken to taste.

\section{Sample size and statistical analysis}

Power analysis based on one degree of freedom assumed from a two-by-two contingency table equated to a sample size of 35 to give a calculated power value of 0.8 . Thirty-five tests were undertaken in accordance with this, and following randomisation with computer-generated random allocation, 20 were allocated to the saccharin group with 15 to placebo. Based on the randomisation process, the nebulised solution, either placebo or saccharin, was prepared by a separate investigator and given to the primary investigator who was blinded.

Statistical analysis was performed using IBM SPSS Statistics for Windows, version 24 (IBM Corp., Armonk, NY, USA). Chisquare and Student's $t$ test analysis was undertaken. A $p$ value of less than 0.05 was considered significant.

\section{Results}

Chi-square test rejected the null hypothesis. The surgical helmet system did not protect against tasting the nebulised saccharin solution. There was a statistically significant difference between the saccharin and the placebo cohort $(p=0.000000024)$.

Table 1 outlines the observed results used for the chisquare test.

Within the saccharin cohort, $8 / 20(40 \%)$ had a positive taste test with the surgical helmet fan on. Mean time to taste was $123.5 \mathrm{~s}(r=34-200 \mathrm{~s})$. With the fan off, all tasted the saccharin with a mean time to taste of $62.6 \mathrm{~s}(r=20-160 \mathrm{~s})$.

Table 1 Observed results from the saccharin vs. placebo cohorts

\begin{tabular}{lccc}
\hline & Positive taste test & Negative taste test & Total \\
\hline Saccharin cohort & 20 & 0 & 20 \\
Placebo cohort & 1 & 14 & 15 \\
Total & 21 & 14 & 35 \\
\hline
\end{tabular}


Student's $t$ test showed a statistically significant difference in the mean time to taste $(p=0.049)$. Figure 1 shows the flow chart of these measured outcomes.

\section{Discussion}

Using saccharin taste as a validated surrogate marker, the results from this study demonstrate that the current surgical helmet fan filters do not protect against aerosol borne particulate $(p<0.0001)$ and therefore would not provide protection against respiratory viruses such as COVID-19. These findings corroborate a previous study based on six subjects published in 2004, which showed that the Stryker T4 and Stackhouse FreedomAire helmet-hood filters alone were deemed not sufficient to protect against transmission of SARS [18].

Guidelines developed by the International Consensus Group on resuming elective orthopaedic surgery following the COVID-19 pandemic advise that whilst surgical helmets should not be used as primary protection against airborne diseases, if they are mandated by hospital standard protocols for total joint arthroplasty, their continued use should be allowed [12]. In this study, with the surgical helmet fan turned on, $40 \%(8 / 20)$ were able to taste the saccharin compared with $100 \%(20 / 20)$ with the fan turned off. Moreover, there was a statistically significant increase in the mean time to taste with the helmet fan turned on (123.5 s) as compared with when the fan was off (62.6 s) $(p=0.049)$. This study confirms that the surgical helmet system does not provide protection against aerosolised particulate. When operating on suspected or confirmed COVID-19 infected patients, the detection of gaseous chemicals and particulate, such as the exhaust from diathermy, can indicate inadequate fit of a respirator mask and necessitate reposition or change. Olfactory cues may well be missed with a surgical helmet system on and therefore place the surgeon at risk. As such the authors would advise against using these helmet systems in restarting elective orthopaedic care whilst the risk of COVID-19 remains high.

In addition, there is potential further risk with the use of a fan system surgical helmet in drawing in the air from directly above the operating field and patient. This may well increase the concentration of airborne droplets within the surgical hood, again increasing exposure risk to the surgeon. Retrofitting the surgical helmet fan to incorporate a compatible filter could provide additional protection and has been designed by the Duke University Medical Centre group using a 3D printed adaptor for the Stryker Flyte Helmet System [10]. In their design, Pall BB50T breathing system filters, usually used to provide filtration protection against aerosol and airborne pathogens in mechanical ventilator systems, were incorporated into the SSHS [10]. It is suggested that this could be potentially used instead of respirator masks if resources are scarce. However, this could also be used in addition to the mask and provide possible solutions to the issues outlined in this study.
Fig. 1 Flow diagram outlining results of saccharin testing

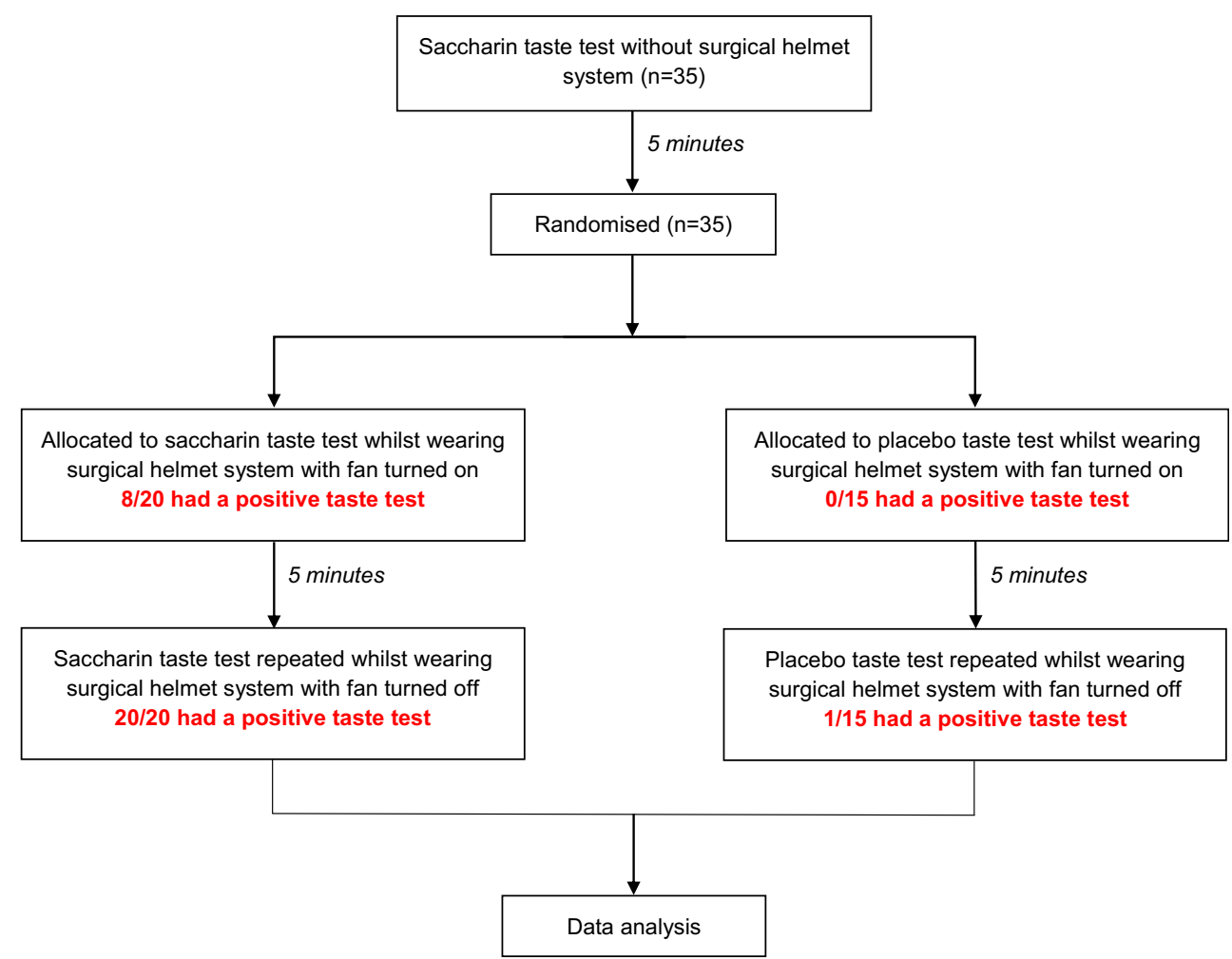


This study exhibits several strengths but also had some limitations. The helmet systems were tested using a validated method of fit testing. As such the saccharin taste test can be considered a surrogate marker for aerosol-borne viruses. The method of randomisation ensured that both participants and investigators had no knowledge of whether participants were receiving saccharin solution or placebo, allowing the use of placebo inhalation as an accurate control. The study was powered appropriately to ensure statistical significance was not due to chance.

Whilst this study did not employ a quantitative fit testing modality, it is of significant clinical relevance and adds to the evidence base for PPE during the reintroduction of elective orthopaedic surgery.

Further studies are required to delineate whether surgical helmets, as they exist currently, increase the amount of particulate flowing through the system as compared with if no helmet was worn. Validation of solutions such as the retrofitted $3 \mathrm{D}$ printed fan filter is also required [10].

\section{Conclusion}

This double-blinded randomised controlled study demonstrates that surgical helmet systems are not effective in preventing entry of respiratory droplets and hence do not provide protection from COVID-19. Furthermore, they may increase risk to the surgeon when operating on patients with possible COVID-19 infection due to the fan masking the ability to notice respirator mask leaks. As such, the authors would therefore recommend against their routine use with the reintroduction of elective orthopaedic services during the COVID-19 pandemic.

Data availability The study performed did not undertake a health-related intervention as defined by the ICMJE, using a surrogate marker instead. As such, registration in a public trials registry does not apply.

\section{Compliance with ethical standards}

Conflict of interest The authors declare that they have no conflict of interest.

Ethics approval Local IRB permission was gained for this study. The study was performed in accordance with the ethical standards as laid down in the 1964 Declaration of Helsinki and its later amendments.

Consent to participate Informed consent was obtained from all individual participants included in the study.

Consent for publication All participants gave informed consent for publication of the study

\section{References}

1. Sohrabi C, Alsafi Z, O'Neill N, Khan M, Kerwan A, Al-Jabir A, Iosifidis C, Agha R (2020) World Health Organization declares global emergency: a review of the 2019 novel coronavirus (COVID-19). Int J Surg (Lond, Engl) 76:71-76. https://doi.org/ 10.1016/j.ijsu.2020.02.034

2. Zhou M, Zhang X, Qu J (2020) Coronavirus disease 2019 (COVID-19): a clinical update. Front Med 14:126-135. https:// doi.org/10.1007/s11684-020-0767-8

3. Jessop ZM, Dobbs TD, Ali SR, Combellack E, Clancy R, Ibrahim N, Jovic TH, Kaur AJ, Nijran A, O'Neill TB, Whitaker IS (2020) Personal protective equipment (PPE) for surgeons during COVID19 pandemic: a systematic review of availability, usage, and rationing. Br J Surg. https://doi.org/10.1002/bjs. 11750

4. Chang Liang Z, Ye Chong MS, Sim MA, Lim JL, Castañeda P, Green DW, Fisher D, Ti LK, Murphy D, Po Hui JH (2020) Surgical considerations in patients with COVID-19: what orthopaedic surgeons should know. J Bone Joint Surg Am. https://doi.org/10.2106/jbjs.20.00513

5. Thomas JP, Srinivasan A, Wickramarachchi CS, Dhesi PK, Hung YM, Kamath AV (2020) Evaluating the national PPE guidance for NHS healthcare workers during the COVID-19 pandemic. Clin Med (Lond, Engl). https://doi.org/10.7861/clinmed.2020-0143

6. WHO (2020) Rational use of personal protective equipment for coronavirus disease (COVID-19) and considerations during severe shortages. In

7. Young SW, Zhu M, Shirley OC, Wu Q, Spangehl MJ (2016) Do 'surgical helmet systems' or 'body exhaust suits' affect contamination and deep infection rates in arthroplasty? A systematic review. J Arthroplast 31:225-233. https://doi.org/10.1016/j.arth.2015.07.043

8. Singh VK, Hussain S, Javed S, Singh I, Mulla R, Kalairajah Y (2011) Sterile surgical helmet system in elective total hip and knee arthroplasty. J Orthop Surg (Hong Kong) 19:234-237. https://doi. org/10.1177/230949901101900222

9. (1996) Surgical helmet systems. Health Dev 25:116-145

10. Erickson MM, Richardson ES, Hernandez NM, Bobbert DW 2nd, Gall K, Fearis P (2020) Helmet modification to PPE with 3D printing during the COVID-19 pandemic at Duke University Medical Center: a novel technique. J Arthroplast. https://doi.org/10.1016/j. arth.2020.04.035

11. FDA (2020) Medical gowns. In

12. Parvizi J, Gehrke T, Krueger CA, Chisari E, Citak M, Onsem S, Walter WL, Abdelaziz H, Abolghasemian MN, Aboltins C, Maskari SM, Baldini A, Barnes CL, Basso T, Belden K, Benazzo F, Bhandari M, Bolognesi MP, Bosco JA, Zhou Y (2020) Resuming elective orthopaedic surgery during the COVID-19 pandemic: guidelines developed by the International Consensus Group (ICM). J Bone Joint Surg 1. https://doi.org/10.2106/JBJS.20.00844

13. FDA (2014) Stryker Flyte hybrid hoods. In

14. Mullins HE, Danisch SG, Johnston AR (1995) Development of a new qualitative test for fit testing respirators. Am Ind Hyg Assoc J 56:1068-1073. https://doi.org/10.1080/15428119591016278

15. Lawrence RB, Duling MG, Calvert CA, Coffey CC (2006) Comparison of performance of three different types of respiratory protection devices. J Occup Environ Hyg 3:465-474. https://doi. org/10.1080/15459620600829211

16. OSHA (2004) OSHA-accepted fit test protocols. In

17. HSE (2016) Health and safety executive 282/28: respiratory protective equipment. In

18. Derrick JL, Gomersall CD (2004) Surgical helmets and SARS infection. Emerg Infect Dis 10:277-279. https://doi.org/10.3201/ eid1002.030764

Publisher's note Springer Nature remains neutral with regard to jurisdictional claims in published maps and institutional affiliations. 